FACTA UNIVERSITATIS

Series: Mechanical Engineering Vol. 16, N 3, 2018, pp. 321 - 335

https://doi.org/10.22190/FUME180823032G

Original scientific paper

\title{
CUSTOMIZATION OF ELECTROSPINNING FOR TISSUE ENGINEERING
}

\author{
UDC 678.3:66.095.26
}

\author{
Nenad Grujović ${ }^{1}$, Fatima Živić ${ }^{1}$, Matthias Schnabelrauch ${ }^{2}$, \\ Torsten Walter ${ }^{2}$, Ralf Wyrwa ${ }^{2}$, Nikola Palić ${ }^{1}$, Lazar Ocokoljić ${ }^{1}$ \\ ${ }^{1}$ University of Kragujevac, Faculty of Engineering, Kragujevac, Serbia \\ ${ }^{2}$ INNOVENT e. V., Biomaterials Department, Jena, Germany
}

\begin{abstract}
This paper deals with two electrospinning technologies: the melt electrospinning with a customized jet head, adapted from the fused deposition modeling (FDM) 3D printer, in comparison with the standard solution electrospinning, aiming at fabrication of tissue engineering scaffolds. The resulting fibers are compared. The influence of the collector properties on those of the fabricated scaffold is investigated. The resulting electrospun fibers exhibit different characteristics such as morphology and thickness, depending on the technology. The micro-fibers are produced by the melt electrospinning with an inbuilt $3 D$ printer jet head, whereas the solution electrospinning has produced nano- and micro-fibers. The scaffolds fabricated on the rotating drum collector exhibit a more ordered structure as well as thinner fibers than those produced on the stationary plate collector. Further investigations should aim at fabrication of porous hollow fibers and tissue engineering scaffolds with controlled porosity and properties.
\end{abstract}

Key Words: Melt Electrospinning, Solution Electrospinning, 3D Printing, Micro-fibers, Nano-fibers

\section{INTRODUCTION}

The spinning process was patented by Anton and Formhals (1934) who described an experimental setup for the production of polymer filaments using an electrostatic force. Electrospinning represents a process for fabrication of polymer nano-fibers by using an electrostatically driven jet of polymer solution or polymer melts [1]. Since 1994, when this technology was put to practical use, electrospinning has been recognized for its ability

Received August 23, 2018 / Accepted November 23, 2018

Corresponding author: Fatima Živić

University of Kragujevac, Faculty of Engineering, Sestre Janjić 6, 34000 Kragujevac, Serbia

E-mail: zivic@kg.ac.rs 
to fabricate nano- and micro-fibers in different forms and of different morphologies [2]. The human extracellular matrix contains submicron fibers and the samples created by electrospinning can closely resemble that network, with a high-specific surface area, which is very important for many applications. Electrospinning has attracted great attention in biomedicine mainly due to: versatility of the technology, ease of use, low cost of the custom made devices, and especially because it can fabricate diverse forms of nanoand micro-fibers [3]. These fibers have been used for tissue engineering scaffolds, to provide different functions [3, 4]; possible fiber structures, morphologies and properties are widely investigated. Electrospun scaffolds can provide a conductive microenvironment for the selected cells thus enhancing tissue regeneration $[4,5]$. There is evidence that electrospun biomaterial samples exhibit rather good mechanical stress resistance and enhance adhesion of cells while their differentiation and proliferation due to the fiber network structure resemble human tissue [6].

The advantages of electrospinning enable its use in different biomedical applications, such as drug release and biotransformation, soft tissue implants and wound healing [7]. Electrospun nanofibers as a drug delivery system show several benefits such as easy modulation of the drug release profile depending upon the properties of the used polymer or polymeric blends, such as antibiotics-loaded nanofibers as wound dressing materials [8]. Two main methods are widely used (solution and melt electrospinning); the influence of the technological parameters on the properties of the final samples is studied beside input materials as well as possible fiber fillers since the complexity of intermixed influences still prevents this technology from wide practical applications [9, 10]. Thakkar and Misra [8] investigated the melt and the solution electrospinning methods for producing curcumin-loaded poly (PCL) fibers in order to find difference between those two methods in characterization and drug release. The results showed that the influence of the curcumin was not the same in both of these electrospinning techniques, thus indicating the necessity to consider the production parameters, beside material properties of the starting polymer. In this case [8], variation of the curcumin content did not change the morphologies of the melt electrospun fiber. However, for the solution electrospun fiber, it resulted in a different set of fiber diameters due to the influence of the curcumin content on the jet balance. A different production technology resulted in a different drug release rate with the same input polymer material.

Additive manufacturing has emerged as the groundbreaking technology affecting many areas due to its possibility to fabricate versatile forms of structures. Fabrication of complex 3D structures also by combining several materials is especially important in tissue engineering [11] even though 3D printing is still under development regarding available materials [12]. Additive biomanufacturing is an important area of research pertaining to tissue regeneration [13], control of fiber diameter and micromechanics [14] or distribution and size of pores within fabricated scaffolds for implantable medical devices [15]. Furthermore, combination of 3D printing and electrospinning has been investigated in fabrication of vascular grafts [16], complex functionalized three-dimensional structures [17] and three-dimensional micro-patterns within 3D structures [18]. Electrical instabilities exhibited in the solution electrospinning can be avoided by using melt materials and 3D printing. In the solution electrospinning, the produced fibers randomly fall onto the collector under the influence of the electric field due to these instabilities, thus preventing precise control of the resulting fiber mat structure. Furthermore, the solvents often exhibit toxicity which is not related to melt materials. The melted material does not exhibit these electrical 
instabilities and 3D printing additionally enables different shapes, structures, even the complex 3D structures to be easily fabricated. This is of the utmost importance in tissue engineering and mimicking of natural tissues. A significant area of application is the production of highly porous hollow fiber membranes whereas the electrospinning technology is modified by using different jet heads and collectors [19, 20]. A high porosity scaffold $(83.5 \%)$ was fabricated by using a porous supporting tube as the collector for nanofibers [19]. Biomimetic scaffolds were fabricated by using a complex collector with a stationary cylindrical hollow piece and a mobile internal cylinder which enabled circumferential and radial alignment of fibers [20]. Some recent applications of 3D printing are for production of complex shapes of collectors in order to enable novel shapes of electrospun fibrous mats [21], multiplexed electrospinning sources [22], structuring of scaffolds [23], or planar arrays of electrospinning emitters [24]. Development of different electrospinning techniques (meltand solution- electrospinning, force-spinning, melt-blowing, flash-spinning, spinning of bicomponent fibers) has become a hot topic due to versatile applications of micro- and nanofibers (tissue engineering, sensors, clothing, membranes for filtration systems, fillers in composite structures, coatings, etc.) [25].

Materials used in electrospinning are mainly polymers but other materials have been recently investigated such as ceramics. Nanofibers made of ceramic are rather hard to produce due to many limitations of these materials but electrospinning has shown excellent results; this is especially important if wide applications of ceramic nanofibers are considered (membranes in filtration systems, fuel cells, smart textiles for wearable technologies, sensors, catalysts and many more) [26]. Chemical sensors based on functional nanofibers made by electrospinning (e.g. in wearable sensors) are also a significant area of research [27].

An interesting application of this technology is related to piezoelectric polymers for selfpowered sensors [28, 29]. Electrospun fibers of the piezoelectric polymers have been investigated for wearable electronics such as medical wearable active sensors for the monitoring of respiration or other health related parameters (vital signs, walking speed, gesture detection, or detection of vocal cords vibration, etc.). Nanofibers of the piezoelectric polymers have been further used in fabrication of smart (functional, active) textiles for a wide range of wearable sensors, such as sensors for vital signs monitoring [30], pressure sensors [31], and tactile sensors [32]. These types of sensors are lightweight and have very high sensitivity thus representing very significant elements in many different applications. For example, these capacitive pressure sensors can detect a water droplet down to $7 \mathrm{mg}$ weight, which makes them highly suitable for wearable electronics in medical applications [31]. Piezoelectric smart fabrics can provide pressure and force sensing, motion and ultrasonic sensing; special yarns have been investigated [33, 34]. For example, these smart fabrics, with embedded electrospun piezoelectric nanofibers, are able to detect even small amounts of ammonia $\left(\mathrm{NH}_{3}\right)$ very fast and they exhibit rapid recovery afterwards [34].

This paper elaborates upon design elements of two electrospinning technologies: the melt electrospinning with a customized jet head, adapted from fused deposition modeling (FDM) 3D printer, in comparison with the standard solution electrospinning, and resulting differences in electrospun fibers, aiming at fabrication of tissue engineering scaffolds. The influence of the collector properties on the fabricated scaffold properties was also investigated. 


\section{EXPERIMENTAL SETUP}

\subsection{Materials and fabrication method}

Three different electrospinning setups are used: the melt electrospinning with an adapted head of the fused deposition modeling (FDM) 3D printer and the standard solution electrospinning with two types of collectors (a stationary plate collector and a rotating drum collector). The nozzle tip orifice at the melt spinning with a FDM jet head has diameter of $0.3 \mathrm{~mm}$. For the solution electrospinning, the conventional $1 \mathrm{ml}$ syringe with a blunt tip needle $(0.603 \mathrm{~mm}$, inner diameter of the needle) is used. The needle temperature is considered to be the same as the ambient temperature since it is not significantly heated due to a large distance of the needle from the pump which is also not heated.

Biodegradable Polycaprolactone (PCL) is used for the melt electrospinning and FP2 Fluoropolymer, $15 \mathrm{wt} \%$ in 1 part DMAc (Dimethylacetamide) and 6 parts Acetone are used for solution electrospinning with a plate collector; PLGA: PLG8523, Poly-lactide (co-glycolide), $8 \mathrm{wt} \%$ in HFIP (Hexafluoroisopropanol).

Parameters of different electrospinning methods are as follows:

- Melt electrospinning with a plate collector: voltage on the plate collector of $30 \mathrm{kV}$; distance from the nozzle tip to the collector surface of $13 \mathrm{~cm}$.

- Solution electrospinning with a plate collector: voltage of $30 \mathrm{kV}$; polymer solution flow rate of $1.5 \mathrm{ml} / \mathrm{h}$; distance from the needle tip to the collector surface of $200 \mathrm{~mm} ; 120$ $\mathrm{mm}$ diameter of the disk above the needle; and $25^{\circ} \mathrm{C}$ temperature within the electrospinning chamber. $0.5 \mathrm{ml}$ of the polymer solution is used as the starting material.

- Solution electrospinning with a drum collector: voltage of $30 \mathrm{kV}$, polymer solution flow rate of $1.5 \mathrm{ml} / \mathrm{h}$; air gap at the syringe/collector distance of $250 \mathrm{~mm}$ and $25^{\circ} \mathrm{C}$ temperature within the electrospinning chamber; $0.5 \mathrm{ml}$ of the polymer solution is used as the starting material. Five different drum rotation speeds are studied: 250, $500,750,1000 \mathrm{rpm}$.

\subsection{Electrospinning technology}

There are two basic types of electrospinning devices, namely those using: 1) the melt electrospinning technique, where the polymer wire flows through the nozzle and melts thus making the polymer fiber that falls down to the collector, and 2) the solution electrospinning technique with a liquid polymer mixture as the starting material that flows through the syringe with a needle. There is a difference between the devices where the fiber is fabricated via melting the polymer through the nozzle, and the other type where the fiber is obtained from a homogenous polymer solution. These two techniques differ in sample characteristics such as morphology, thickness of electrospun fibers, transparency, etc. Selection of the appropriate technique depends on the material used. For example, some polymers worsen their properties when exposed to high temperatures hence the melting technique is not applicable. Also, fiber thickness is determined by different techniques.

Special attention should be devoted to the adequate polymer starting form, depending on the electrospinning process that is used. In the case of the solution electrospinning, voltage is brought to the needle and the liquid polymer is put into the syringe, which is further mounted on the element that pushes the syringe plunger with precisely defined speed. The quantity of the polymer melt that is expelled through a discharge orifice at the open end of the tube and 
into the needle is determined by the linear speed of the plunger, as setup at the beginning of the process. In the case of the melt electrospinning, voltage is brought to the collector and the polymer wire is brought to the nozzle and fed into it by the step motor with predefined speed. The polymer wire melts inside the nozzle whereas the temperature within the nozzle can be controlled and is setup according to the polymer type.

In both of these methods, the polymer droplet created at the tip of the needle/nozzle is further electrified and elongated to form a thin fiber, as it falls through the electric field. The distance between the needle/nozzle tip and the collector as well as the value of electric field, determine the structure and the thickness of the electrospun fiber. The speed of the polymer passing through the needle/nozzle also has significant influence on the final fibers properties.

One of the main issues in electrospinning is poor repeatability of results [3,9]. It is not possible to predict the results with high certainty even with the same materials and process parameters; hence it is necessary to constantly monitor the process. In the case when the distance between the needle/nozzle and collector surface is too large, or the electric field is not well adjusted, the fibers can fly away from the projected collecting zone on the collector and fly randomly around the chamber. Very often, different final fiber mats are produced with the same process parameters and materials. On the other hand, even small variations in fiber morphologies and structure can significantly affect final scaffold function, especially for its further nano scale responses. If the polymer solution density is not well adjusted, it can result in polymer drops directly falling on the collector without forming fibers. In the case of high viscosity polymers, such issue is not present. However, high viscosity of polymers can produce random abrupt stopping and starting of the pump which pushes the polymer solution through the syringe into the needle, thus resulting in excessive quantities of the polymer solution fed into the syringe. The issue of the possibility of such large droplets falling onto the collector and damaging the fiber mat is solved by manual removal of the droplets from the needle by using a plexiglas rod. This is one common reason for continuous monitoring of the electrospinning process. Sometimes, in the case of very high viscosity polymer solutions (such as honey viscosity), the syringe can bend under the large force exerted upon it by such "thick" fluid flow.

\subsubsection{Melt electrospinning}

In the case of the melt electrospinning technique, a polymer wire flows through the nozzle and melts, thus making a polymer fiber that falls down to the collector (Fig. 1). This concept with the polymer wire moving and melting through the nozzle represents the same concept as used in the 3D printer. The wire is pushed through the nozzle by a step motor while the controlled heater warms up the nozzle. 3D printer heads can be used in combination with the electrospinning chamber and such custom made devices for production of fibrous scaffolds have been recently investigated [13, 14, 15].

One such electrospinning device was designed and presented in this paper. A nozzle hole diameter through which the wire is melted is around $0.3 \mathrm{~mm}$. Voltage brought to the collector is of order of several $10 \mathrm{kV}$. In that way, a strong electrical field is formed, which initiate the attraction of the polymer fiber by the collector. Delivering of voltage to the collector is done by an electrical transformer. The main elements of the melt electrospinning device are given in Fig. 1a. 

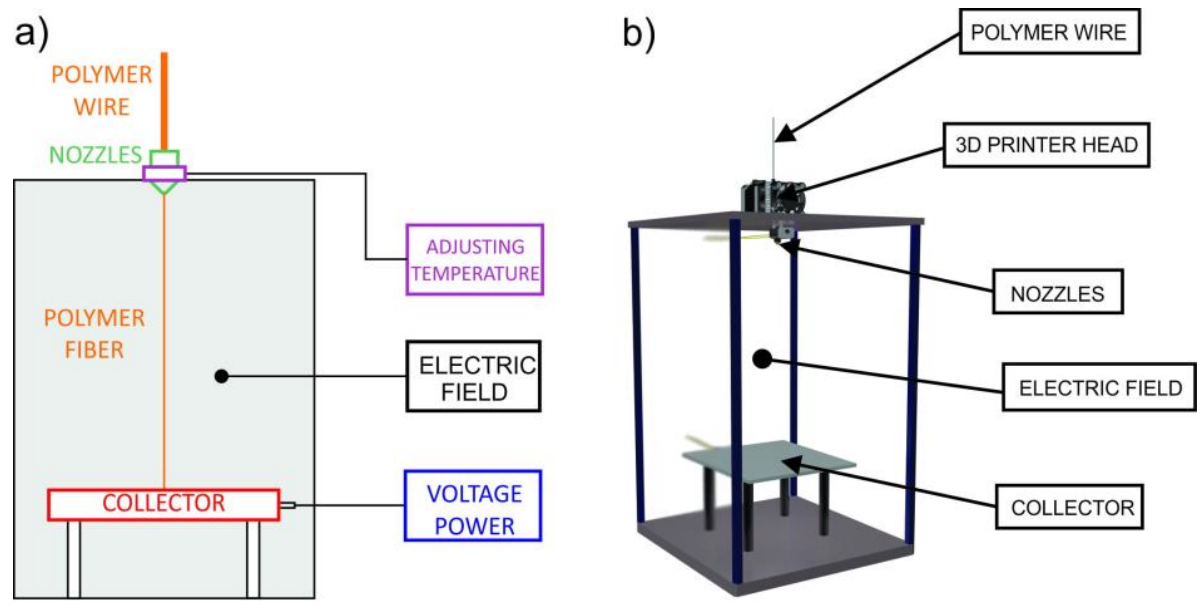

Fig. 1 Main elements of the melt electrospinning device:

a) schematic representation of the process, b) design elements

For this type of device, it is very important to adequately setup the parameters according to the specific test. If the melting temperature is too low, the material will melt but it will also stay too viscous to extract the fiber. On the other hand, if the temperature is too high, the material will burn. This is especially important in the case of different polymer mixtures (such as mixtures containing antibiotics) because improper work parameters can have a negative effect on some of the polymer properties. Possible issue for this type of device is related to the wire that is used as starting material and which is made by using an extruder thus raising technology costs. One possible design of the melt spinning device is given in Fig. $1 \mathrm{~b}$.

\subsubsection{Solution electrospinning}

The solution electrospinning technique uses a liquid polymer mixture as the starting material. The material is in the syringe connected to the needle which is located within the electrospinning device. The needle is within the bearing which is situated in the metal disk where voltage is delivered. The main elements of the solution electrospinning device are given in Fig. 2a. Voltage is delivered to the needle (via the metal disk) unlike the previous design where voltage is delivered to the collector. The size of the disc directly influences the size of the collector area where the sample will be created (the greater size of the disk, the greater size of the collector area). Amount of the polymer mixture which goes through the needle is regulated by the module in which the syringe is installed and the flow parameters are setup. Depending on the input parameters of the process, the polymer solution is pressed through the syringe. It is important that the flat top needle is used to prevent accumulation of fibers at only one side of the collector as would be in the case of the medical bevel needle. One possible design of the solution electrospinning device is given in Fig. $2 b$. 
a)

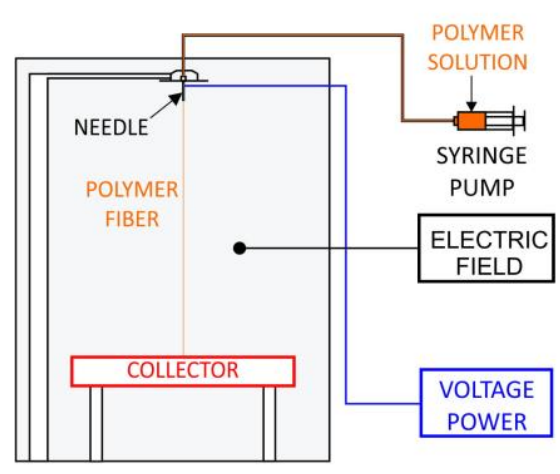

b)

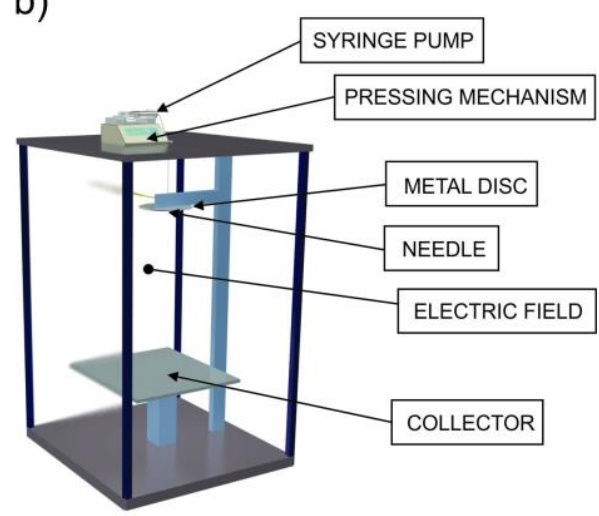

Fig. 2 Main elements of the solution electrospinning device:

a) schematic representation of the process, b) design elements

\subsubsection{Customization of electrospinning device}

An essential element of the electrospinning device is the electrospinning head (or jet head). Different jet heads are used in the melt and the solution electrospinning: a nozzle (to provide wire or melt feeding) or a syringe (to provide solution flow). Different designs of the jet head are investigated aiming to provide efficient material feed, including composites [22, 24]. Electrospun fibers have been investigated for different biomedical applications, such as drug delivery. Composite nanofibers (or core - shell systems) represent a novel direction of research in electrospinning techniques, based on the custom design of jet head and collectors. These composite nanofibers comprise the core made of one material, covered with a thin layer (shell) made of different material. Special design of the element used in electrospinning device (a multi-jet electrospinning head) which introduces two different polymers into the electric field is shown in Fig. 3. The needle (with one type of polymer solution - inner fluid) is located within the nozzle (with another type of polymer solution outer fluid). Altogether core - shell output droplets and subsequent fibers are fabricated within the electric field. Diameter of the core zone and thickness of the shell cover are regulated and controlled by the speed of simultaneously feeding the polymers into the needle and the nozzle.

Different combinations of custom-design jet heads and collectors can produce versatile final fiber structures, such as hierarchically structured fibers [35], nanofibers with $\Omega$-shaped shell [36], or 3D nanostructured scaffold with $99.98 \%$ porosity [37]. Investigation of different designs of device elements and literature review pointed out that the melt electrospinning should be used for production of micro-fibers, whereas the solution electrospinning can efficiently produce nano-fibers. One of the special designs of the jet head, as given in Fig. 3, can be used to produce core-shell fiber structures. Additional customization of the collector shapes, numbers and motion, can produce different final shapes of the fiber mat. Depending on the final application, several different collectors can be used, some of which are shown in Fig. 4. 


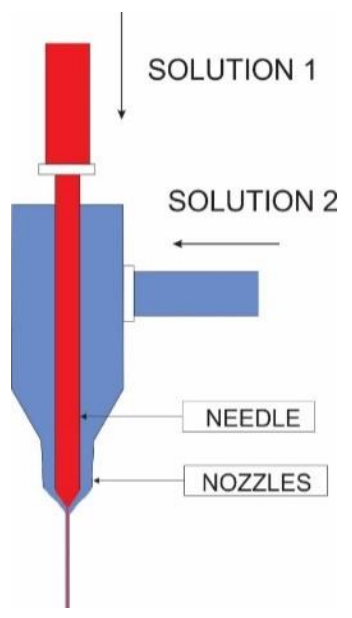

Fig. 3 Multi jet electrospinning head used for composite nanofibers production

Another element of the electrospinning device, that can be custom shaped, is the collector where the fibers are collected after their forming within the electric field. A fine porous scaffold (fiber mat) is fabricated on the collector surface. The type of the collector used in the process has significant influence on the distribution of the fibers, and further on the porous scaffold sample. Different types of collectors can be used for electrospinning process and new designs have been investigated, especially in combination with multiple electrospinning sources [22, 24]. High voltage brought to the collector (or the needle) creates a very strong electric field which elongates the droplet and creates fibers that are further attached to the collector. The sample is formed on the surface layer of the collector which is different from the bulk material of the collector in order to better control the final sample output.

Plate collectors and rotating (drum) collectors are standard forms whereas several new shapes and types of movements are currently under investigations [20, 22, 37]. Plate collectors can be fixed with no motion, or they can exhibit motion, such as simple translational motion in horizontal plane (Fig. 4a). Translational motion produces a larger sample, whereas uniform speed results in homogenous density. Speed and trajectory can be predefined and controlled. Variable speed produces different thickness distribution of the sample along the sample. The spots where the collector is fixed by screws can sometimes exhibit thicker samples due to an increased quantity of metallic material in these spots thus resulting in accumulation of fibers. Usually, a glass layer is installed on the plate collector in order to allow an easier control of the sample. Design of the drum collector is given in Fig. 4b. It rotates during electrospinning, thus creating a scaffold in the form of a round hollow pipe. Rotation speed of the collector directly affects the thickness of the scaffold. Aluminum foil is usually fixed on the collector's surface to enable removal of the sample afterwards. Also, the collector can be designed to create tube samples, like the one shown in Fig. 4c. This collector operates similarly to a drum collector. The cylinder diameter is much smaller

a)

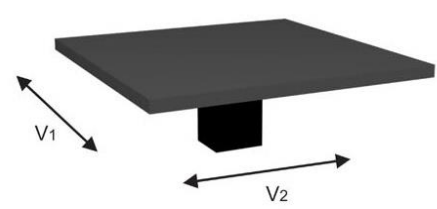

b)

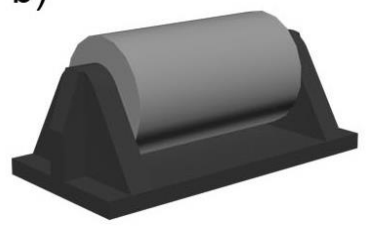

c)

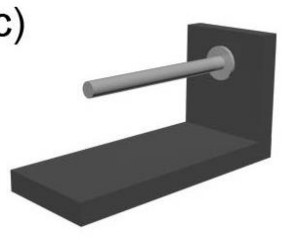

Fig. 4 Different types of collectors: a) plate collector with directions of translational motion (variable speed), b) drum collector, c) design of the tube-form sample collector 
than at the drum collector, thus resulting in rapid accumulation of fibers. This type of the collector has been used to produce scaffolds made of biodegradable polymers aiming at blood vessels repair. An electrospun scaffold can be used for coating a damaged vessel until its healing.

\section{RESULTS AND DISCUSSION}

A new approach to the custom design of the electrospinning device is to combine 3D printing and electrospinning techniques into one device or to use it parallel to each other in the synchronized manner $[16,17,18]$. In our work, the head of the fused deposition modeling (FDM) 3D printer was used instead of the standard melt electrospinning jet head. The main elements of such jet head are shown in Fig. 5a. One fiber mat sample made at the adapted electrospinning device is shown in Fig. 5b. This device setup has been used to overcome the recognized issues in fabrication of drug-impregnated fibers for drug delivery systems. Drugs in the pre-fabricated drug-loaded wire can be damaged by a high melt temperature within the jet head in the case of the standard melt electrospinning device. The jet head adapted from the 3D printer also melts the wire and produces an initial polymer drop to enter the force field but it exhibits adequate temperatures thus preserving initial properties of the drug, which is further transferred into the electrospun fibers. A stepper motor is used to feed the wire into the nozzle where it melts. This device setup is well suited for drug delivery applications where larger fibers (micro to macro fibers) are needed. The wire that was fed into the adapted jet head is custom made of PCL biodegradable polymer, by using a wire extruder.
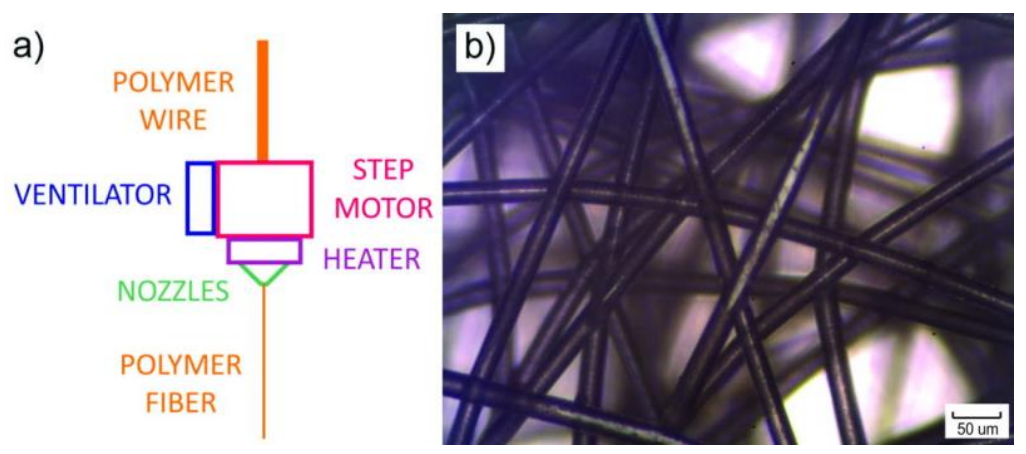

Fig. 5 a) Main elements of the jet head in melt electrospinning device, by using adapted 3D printer head and b) sample produced at such adapted device (PCL micro-fibers)

Thin polymer fibers are made during electrospinning under the influence of electric force which elongates the initial polymer droplet during its falling from the needle/nozzle tip to the collector. Fibers are continuous until the polymer is fed to the needle/nozzle and electric force is acting on it. In general, final fibers are randomly distributed over the collector's surface. Thickness and morphologies of the fibers are different if the melt- and the solution- electrospinning are compared. The melted polymer has higher viscosity than its solution and the nozzle orifice is larger than the needle tip. Accordingly, the thickness 
of the fibers made by the melt electrospinning tends to be larger (micron scale) than those made by the solution electrospinning (nano to micron scale). FP2 Fluoropolymer fibers made by using the solution electrospinning with a plate collector (Fig. 4a) are shown in Fig. 6.
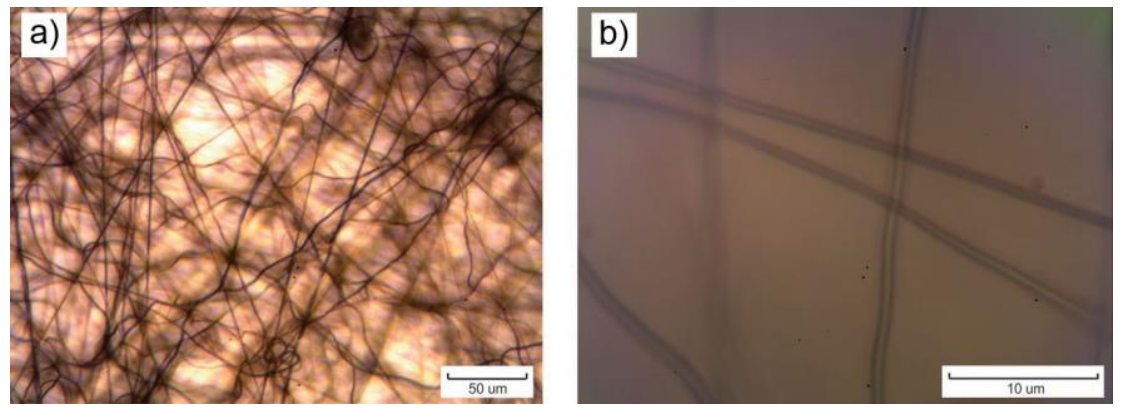

Fig. 6 FP2 Fluoropolymer fibers produced by solution electrospinning, collected on the plate collector, two magnifications

There is a clear difference between the size of the electrospun fibers produced at the solution spinning (Fig. 6) and at the adapted electrospinning device (Fig. 5b). The size of the nozzle tip orifice $(0.3 \mathrm{~mm})$ has a comparable length scale to the standard inner diameter of the syringe needle $(0.603 \mathrm{~mm})$. The adapted setup offers more possibilities for precise tuning of the fiber properties in the case of micro/macro fibers, and, accordingly, of the resulting fibrous scaffold. Our work is fully in accordance with several authors showing that the FDM 3D printer head can be used in the melt electrospinning, especially important for composite fibers impregnated with different materials $[13,14,15]$. The 3D printer head enables a customized input material since we have produced the wire by using the extruder which enables different material combinations, including drug loading of the wire. Fiber diameters and fiber density are under investigation for different applications while the combination of FDM 3D printing with electrospinning can provide good tailoring of properties at the micro/macro levels. Combination of different materials, or synchronized use of additive manufacturing technologies, together with customization of elements in the electrospinning device, have been investigated in order to provide better control and repeatability of the process.

Beside device elements (jet head, type of collector), process parameters significantly influence the resulting electrospun fibers. The change of the collector shape (the drum instead of the plate collector) and rotation speed of the drum collector $(250,500,750,100$ $\mathrm{rpm})$, results in changes of the fiber thickness and their spatial distribution within the scaffold, as shown in Fig 7. 

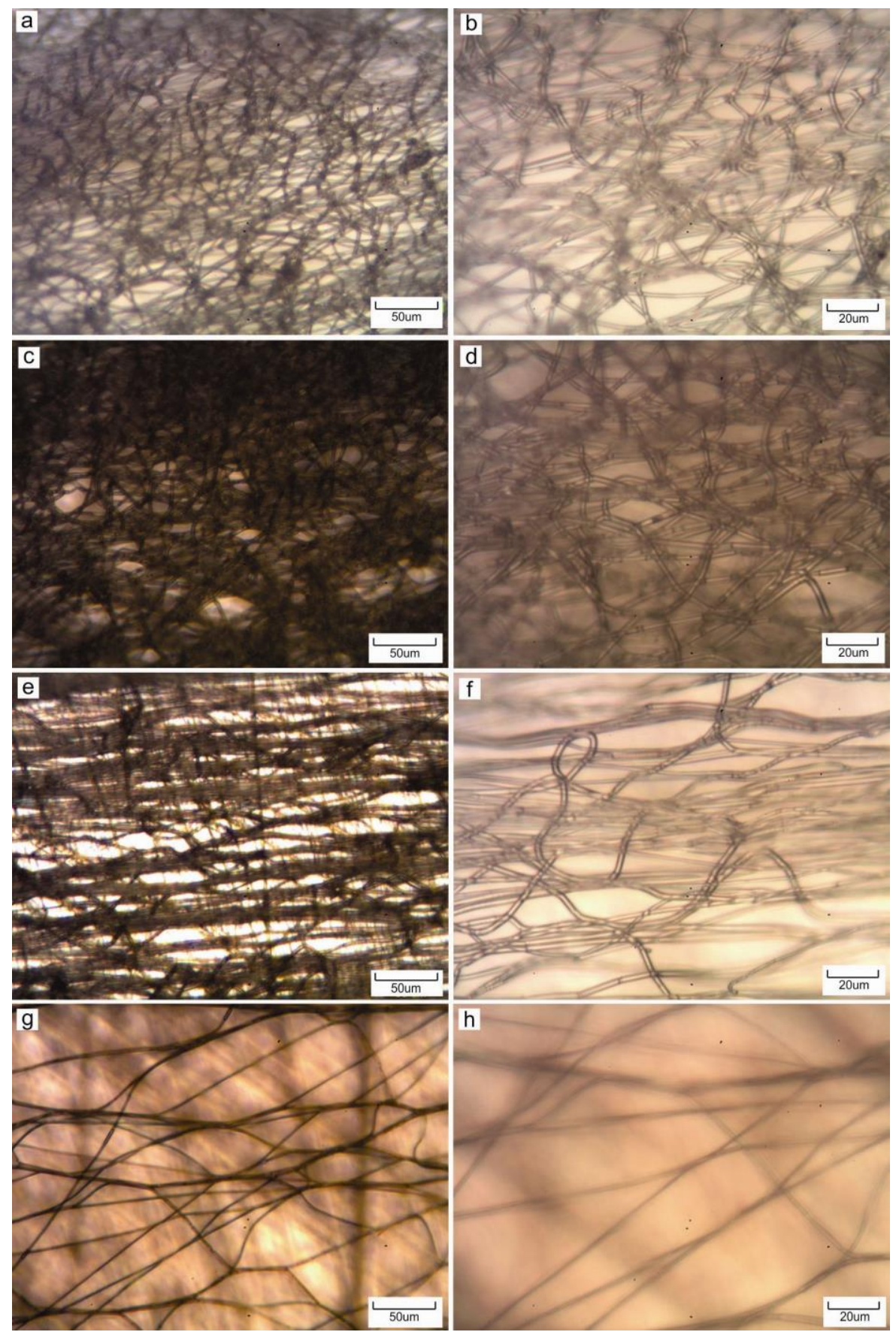

Fig. 7 Diameter of PLGA polymer fibers fabricated by solution electrospinning, depending on the rotation speed: a), b) $2.4 \mu \mathrm{m}, 250 \mathrm{rpm}$; c), d) $2.2 \mu \mathrm{m}, 500 \mathrm{rpm}$; e), f) $2.2 \mu \mathrm{m}$, $750 \mathrm{rpm}$; g), h) $2 \mu \mathrm{m}, 1000 \mathrm{rpm}$ 
The major influence of the rotation speed change is on the thickness of the produced fibers. The influence of the rotation speed of the drum is obvious, as shown in Fig 7. Increase of the rotation speed produces thinner fibers. Fiber diameter was approx. $2.4 \mu \mathrm{m}$ at $250 \mathrm{rpm}$, down to $2 \mu \mathrm{m}$ at $1000 \mathrm{rpm}$. The rotating drum twists the fiber additionally, making it thinner, and continually winds the fibers onto the drum, unlike the stationary plate collector. Comparison of the fiber diameter sizes for different electrospinning methods used in this work is shown in Table 1.

Table 1 Comparison of fiber diameter sizes for different electrospinning methods

\begin{tabular}{lc}
\hline Electrospinning method & Fiber diameter, $\mu \mathrm{m}$ \\
\hline Melt electrospinning, material: PCL & 20 \\
Solution electrospinning, plate collector, material: FP2 Fluoropolymer & $0.5-0.8$ \\
Solution electrospinning, drum collector, 250 rpm, material: PLGA & 2.4 \\
Solution electrospinning, drum collector, 500 rpm, material: PLGA & 2.2 \\
Solution electrospinning, drum collector, 750 rpm, material: PLGA & 2.2 \\
Solution electrospinning, drum collector, 1000 rpm, material: PLGA & 2 \\
\hline
\end{tabular}

Comparison of the scaffolds produced on the plate and drum collectors indicate certain differences. The major difference between the fibers and the scaffolds produced by the solution electrospinning with the plate collector to the same technology with the drum collector is the resulting shape of the scaffold, together with different pore spatial distribution within the scaffold and the thickness of the electrospun fibers. The scaffolds produced on the plate collector comprise completely random positioning of the fibers falling upon it. The drum collector rotation influences some degree of ordered positioning of the falling fibers with simultaneous winding of the fibers onto the drum, which is similar to the spinning wheel used for textile yarn production. Fibers are not completely arranged along one line in the direction of rotation, but the scaffold exhibits a much less random structure in comparison with the standard solution electrospun fiber on the plate collector.

Using melt materials like in 3D printing, the electrospun fibers do not exhibit electrical instabilities which are imminent to the solution electrospinning, as one of the main reasons for poor repeatability of results [3]. Accordingly, better control of the resulting scaffold properties can be gained.

An additional advantage of the combination of 3D printing and standard electrospinning is adaptability of the device and its low cost, thus enabling wide investigation of possible tissue engineered scaffold models. For example, combination of layers can be realized, by using two heads alternatively, one after each other, in one electrospinning process. FDM jet head can be used to fabricate one type of fiber layers, upon which a layer produced by solution electrospinning is placed, alternatively many times, thus producing scaffolds comprising two materials (e.g. drug loaded fibers throughout the scaffold made of another material). Customization of the material composition of the input wire can be efficiently performed by using a wire extruder, thus enabling different composites and material combinations. Hence, the drug loaded polymer wire can be prepared at the extruder and enter the FDM jet head of the custom electrospinning device. Our results show that this low cost, simple solution is well suited for production of drug loaded micro-fibers and fibrous scaffolds for tissue engineering (e.g. for wound patches). However, further investigations are needed related to the precise control of all process parameters and especially of their influence on the morphology and structure of the resulting tissue scaffolds. 


\section{CONCLUSIONS}

Electrospinning has gained significant attention as efficient technology for production of nano- and micro-fibers and porous, fibrous structures for tissue engineering scaffolds. Our investigation shows that customization of device elements can influence the properties of final fibers, such as: morphology or thickness, as well as the final mat properties (size, shape, thickness, porosity). It is shown that a combination of 3D printer elements (FDM head) with an electrospinning device is completely suited for fabrication of micro-fibers. A direct possibility derived from using the FDM head as the electrospinning jet head is opening up of possible input material combinations aiming towards hollow and drug loaded fibers. The 3D printer head enables customized input material since the applied wire is produced by using an extruder which enables different material combinations, including drug loading of the wire. It is shown that this is a cost-efficient solution for melt-electrospinning of micro-fibers. There is a clear difference in diameter sizes of such melt-electrospun fibers, in comparison with the standard solution electrospinning. PCL polymer fibers of micro-size diameter (approx. $20 \mu \mathrm{m}$ ) are fabricated by using melt electrospinning with an adapted 3D printer head, whereas the solution electrospinning results in thinner fibers (FP2 Fluoropolymer, 0.5$0.8 \mu \mathrm{m}$ and PLGA, $2-2.4 \mu \mathrm{m}$ ). Fibers and scaffolds produced on the stationary plate and the rotating drum collector are different, respectively, regarding diameter size $(0.5-0.8 \mu \mathrm{m}$, plate collector; $2-2.4 \mu \mathrm{m}$, drum collector) and uniformity of the fibers positioning within the scaffolds. Increase of the rotation speed results in thinner fibers. Further investigation is needed to provide a full process control, and comprehensively understand relations between specific electrospinning technology and process parameters and the morphology and structure of the fabricated fibers and fibrous scaffolds.

Acknowledgements This work is supported by the national projects III41017 and TR35021, financed by the Ministry of Education, Science and Technological Development of the Republic of Serbia for the period of 2011-2018.

\section{REFERENCES}

1. Ramakrishna, S., Fujihara, K., Teo, W.E., Lim, T.C., Ma, Z., 2005, An Introduction to Electrospinning and Nanofibers, World Scientific Publishing, Singapore, 396 p.

2. Yukseloglu, S.M., Sokmen, N., Canoglu, S., 2015, Biomaterial applications of silk fibroin electrospun nanofibers, Microelectronic Engineering, 146, pp. 43-47.

3. Muerza-Cascante, M.L., Haylock, D., Hutmacher, D.W., Dalton, P.D., 2015, Melt electrospinning and its technologization in tissue engineering, Tissue Engineering - Part B: Reviews, 21(2), pp. 187-202.

4. Cui, H., Nowicki, M., Fisher, J.P., Zhang, L.G., 2017, 3D Bioprinting for Organ Regeneration, Advanced Healthcare Materials, 6(1), 1601118.

5. Lanza, R., Langer, R., Vacanti, J., 2007, Principles of Tissue Engineering 3rd ed., Academic Press, Utah, USA, $1244 \mathrm{p}$.

6. Mouthuy, P.A., Ye, H., 2011, Biomaterials: Electrospinning. In: Comprehensive Biotechnology $2^{\text {nd }}$ ed, Murray, M., (Ed), Elsevier, Amsterdam, 5, 12 p.

7. Hall Barrientos, I.J., Paladino, E., Szabó, P., Brozio, S., Hall, P.J., Oseghale, C.I., Passarelli, M.K., Moug, S.J., Black, R.A., Wilson, C.G., Zelkó, R., Lamprou, D.A., 2017, Electrospun collagen-based nanofibres: A sustainable material for improved antibiotic utilisation in tissue engineering applications, International Journal of Pharmaceutics, 531, pp. 67-79.

8. Thakkar, S., Misra, M., 2017, Electrospun polymeric nanofibers: New horizons in drug delivery, European Journal of Pharmaceutical Sciences, 107, pp. 148-167. 
334 N. GRUJOVIĆ, F. ŽIVIĆ, M. SCHNABELRAUCH, T. WALTER, R. WYRWA, N. PALIĆ, ET AL.

9. Wang, X., Xu, Y., Wei, Q., Cai, Y., 2011, Study on technological parameters effecting on fiber diameter of melt electrospinning, Advanced Materials Research, 332-334, pp. 1550-1556.

10. Lian, H., Meng, Z., 2017, Melt electrospinning vs. solution electrospinning: A comparative study of drugloaded poly (E-caprolactone) fibres, Materials Science and Engineering: C, 74, pp. 117-123.

11. Zivic, F., Grujovic, N., Mitrovic, S., Adamovic, D., Petrovic, V., Radovanovic, A., Djuric, S., Palic, N., 2016, Friction and Adhesion in Porous Biomaterial Structure, Tribology in Industry, 38(3), pp. 361-370.

12. Perepelkina, S., Kovalenko, P., Pechenko, R., Makhmudova, K., 2017, Investigation of Friction Coefficient of Various Polymers Used in Rapid Prototyping Technologies with Different Settings of $3 D$ Printing, Tribology in Industry, 39(4), pp. 519-526.

13. Carter, S.-S.D., Costa, P.F., Vaquette, C., Ivanovski, S., Hutmacher, D.W., Malda, J., 2017, Additive Biomanufacturing: An Advanced Approach for Periodontal Tissue Regeneration, Annals of Biomedical Engineering, 45(1), pp. 12-22.

14. Ko, J., Ahsani, V., Yao, S.X., Mohtaram, N.K., Lee, P.C., Jun, M.B.G., 2017, Fabricating and controlling PCL electrospun microfibers using filament feeding melt electrospinning technique, Journal of Micromechanics and Microengineering, 27(2), 025007.

15. Biscaia, S., Dabrowska, E., Tojeira, A., Horta, J., Carreira, P., Morouço, P., Mateus, A., Alves, N., 2017, Development of Heterogeneous Structures with Polycaprolactone-Alginate Using a New 3D Printing System - BioMEDßeta: Design and Processing, Procedia Manufacturing, 12, pp. 113-119.

16. Centola, M., Rainer, A., Spadaccio, C., De Porcellinis, S., Genovese, J.A., Trombetta, M., 2010, Combining electrospinning and fused deposition modeling for the fabrication of a hybrid vascular graft, Biofabrication, 2(1), 014102.

17. Park, S.H., Kim, T.G., Kim, H.C., Yang, D.Y., Park, T.G., 2008, Development of dual scale scaffolds via direct polymer melt deposition and electrospinning for applications in tissue regeneration, Acta Biomaterialia, 4, pp.1198-1207.

18. Rogers, C.M., Morris, G.E., Gould, T.W.A., Bail, R., Toumpaniari, S., Harrington, H., Dixon, J.E., Shakesheff, K.M., Segal, J., Rose, F.R.A.J., 2014, A novel technique for the production of electrospun scaffolds with tailored three-dimensional micro-patterns employing additive manufacturing, Biofabrication, 6(3), 035003.

19. Su, C., Lu, C., Cao, H., Gao, F., Chang, J., Li, Y., He, C., 2017, Fabrication of a novel nanofibers-covered hollow fiber membrane via continuous electrospinning with non-rotational collectors, Materials Letters, 204, pp. 8-11.

20. Stocco, T.D., Rodrigues, B.V.M., Marciano, F.R., Lobo, A.O., 2017, Design of a novel electrospinning setup for the fabrication of biomimetic scaffolds for meniscus tissue engineering applications, Materials Letters, 196, pp. 221-224

21. Paterson, T.E., Beal, S.N., Santocildes-Romero, M.E., Sidambe, A.T., Hatton, P.V., Asencio, I.O., 2017, Selective laser melting-enabled electrospinning: Introducing complexity within electrospun membranes, Proceedings of the Institution of Mechanical Engineers, Part H: Journal of Engineering in Medicine, 231(6), pp. 565-574.

22. García-López, E., Olvera-Trejo, D., Velásquez-García, L.F., 2017, 3D printed multiplexed electrospinning sources for large-scale production of aligned nanofiber mats with small diameter spread, Nanotechnology, 28(42), 425302.

23. Hosseini, S., Khenoussi, N., 2017, Structuring of electrospun nanofiber mats by 3D printing methods, In: Uyar, T., Kny, E., Electrospun Materials for Tissue Engineering and Biomedical Applications: Research, Design and Commercialization, Woodhead Publishing, pp. 73-85.

24. Ponce De Leon, P.J., Hill, F.A., Heubel, E.V., Velásquez-García, L.F., 2015, Parallel nanomanufacturing via electrohydrodynamic jetting from microfabricated externally-fed emitter arrays, Nanotechnology, 26(22), 225301.

25. Nayak, R., Padhye, R., Arnold, L., 2017, Melt-electrospinning of nanofibers, Electrospun Nanofibers, A volume in Woodhead Publishing Series in Textiles, pp. 11-40.

26. Esfahani, H., Jose, R., Ramakrishna, S.C., 2017, Electrospun ceramic nanofiber mats today: Synthesis, properties, and applications, Materials Open Access, 10(11), Article number 1238.

27. Persano, L., Camposeo, A., Pisignano, D., 2017, Advancing the Science and Technology of Electrospinning and Functional Nanofibers, Macromolecular Materials and Engineering, 302 (8), Article number 1700237.

28. Fuh, Y.K., Lee, S.C., Tsai, C.Y., 2017, Application of Highly flexible self-powered sensors via sequentially deposited piezoelectric fibers on printed circuit board for wearable electronics devices, Sensors and Actuators, A: Physical, 268, pp. 148-154. 
29. Liu, Z., Zhang, S., Jin, Y.M., Ouyang, H., Zou, Y., Wang, X.X., Xie, L.X., Li, Z., 2017, Flexible piezoelectric nanogenerator in wearable self-powered active sensor for respiration and healthcare monitoring, Semiconductor Science and Technology, 32(6), Article number 064004.

30. Lee, S., Ahn, Y., Prabu, A., Kim, K., 2013, Piezoelectric Polymer and Piezocapacitive Nanoweb Based Sensors for Monitoring Vital Signals and Energy Expenditure in Smart Textiles, Journal of Fiber Bioengineering and Informatics, 6(4), pp. 369-381.

31. Kim, Y., Jang, S., Kang, B.J., Oh, J.H, 2017, Fabrication of highly sensitive capacitive pressure sensors with electrospun polymer nanofibers, Applied Physics Letters, 111(7), Article number 073502.

32. Lee, H.B., Kim, Y.W., Yoon, J., Lee, N.K., Park, S.-H., 2017, 3D customized and flexible tactile sensor using a piezoelectric nanofiber mat and sandwich-molded elastomer sheets, Smart Materials and Structures, 26(4), 045032.

33. Yang, E., Xu, Z., Chur, L.K., Behroozfar, A., Baniasadi, M., Moreno, S., Huang, J., Gilligan, J., MinaryJolandan, M., 2017, Nanofibrous Smart Fabrics from Twisted Yarns of Electrospun Piezopolymer, ACS Applied Materials and Interfaces, 9(28), pp. 24220-24229.

34. Wu, S., Liu, P., Zhang, Y., Zhang, H., Qin, X, 2017, Flexible and conductive nanofiber-structured single yarn sensor for smart wearable devices, Sensors and Actuators, B: Chemical, 252, pp. 697-705.

35. Yang, G., Li, X., He, Y., Ma, J., Ni, G., Zhou, S., 2018, From nano to micro to macro: Electrospun hierarchically structured polymeric fibers for biomedical applications, Progress in Polymer Science, 81, pp.80-113.

36. Hu, M., Teng, F., Chen, H., Jiang, M., Gu, Y., Lu, H., Hu, L., Fang, X., 2017, Novel S-Shaped Core-Shell Photodetector with High Ultraviolet Selectivity and Enhanced Responsivity, Advanced Functional Materials, 27(47), Article number 1704477.

37. Hejazi, F., Mirzadeh, H., Contessi, N., Tanzi, M.C., Faré, S., 2017, Novel class of collector in electrospinning device for the fabrication of $3 D$ nanofibrous structure for large defect load-bearing tissue engineering application, Journal of Biomedical Materials Research - Part A, 105(5), pp. 1535-1548. 